\title{
AN EXAMPLE IN THE THEORY OF POINTWISE PERIODIC HOMEOMORPHISMS*
}

\author{
DICK WICK HALL $†$
}

A single-valued continuous transformation $T(M)=M$ is said to be pointwise periodic $\ddagger$ provided that for every point $x$ in $M$ there exists an integer $N_{x}$ such that $T^{N_{x}}(x)=x$. The smallest such integer $N_{x}$ (greater than zero) is called the period of $x$ under $T$. For each point $x$ of $M$ the finite subset of $M$ consisting of all the images of $x$ under $T$ is called the orbit of $x$ under $T$.

In most of the familiar examples concerned with this type of transformation the limit $\S$ of every convergent sequence of orbits consists of a set of points all having the same period. In fact the first example in which this is not the case has recently been given by G. E. Schweigert and the author. $\|$ In this paper we combine this example with one found by Ralph Phillips and W. L. Ayres $\Phi$ and then generalize the result to obtain what is probably the first example in which the space is a locally connected continuum while at the same time there exists a sequence of orbits converging to a set $L$ containing points of arbitrary preassigned periods.

We let $r_{1}=1$ and $r_{2}, r_{3}, \cdots$ be the arbitrarily chosen periods for points in $L$. Then we construct a locally connected continuum $M$ in four-dimensional euclidean $(x, y, z, w)$ space and define a pointwise periodic homeomorphism $T(M)=M$ with the following properties:

(a) There exists a convergent sequence of orbits $\left(G_{i}\right)$ under $T$ having a limit set $L$ which for every $i=1,2, \cdots$ contains a free arc every interior point of which has period exactly $r_{i}$.

(b) The closure of every component of $M-L$ is a 2-cell.

* Presented to the Society, November 26, 1938, under the title On pointwise periodic homeomorphisms.

† National Research Fellow in Mathematics.

¥ See Deane Montgomery, Pointwise periodic homeomorphisms, American Journal of Mathematics, vol. 59 (1937), pp. 118-120.

$\S$ The limit superior of a sequence of point sets consists of all points every neighborhood of which contains points from infinitely many sets of the sequence. The limit inferior consists of all points every neighborhood of which contains points from all but at most a finite number of sets of the sequence. If these two sets are identical, their common value is known as the limit of the sequence.

II See D. W. Hall and G. E. Schweigert, Duke Mathematical Journal, vol. 4 (1938), p. 723.

I See a forthcoming article by W. L. Ayres in Fundamenta Mathematicae. The example of Phillips and Ayres shows that a pointwise periodic homeomorphism need not be almost periodic even though the space is a locally connected continuum. 
We begin by constructing the limit set $L$ in the $(x, y)$ plane, and do this by means of polar coordinates $(r, \theta)$. In this plane let $C$ be the unit circle $r=1$, and define an infinite sequence of points $\left(a_{i}\right)$ as follows: $a_{0}=(1,0), a_{i}=(1,[1+(i-1) / i] \pi),(i \neq 0)$. Evidently, $\lim a_{i}=a_{0}$.

If the sequence $\left(r_{i}\right),(i=1,2, \cdots)$, contains only a finite number of terms, we may repeat the last term infinitely many times. Hence we lose no generality in assuming this sequence to be infinite. Let $A_{i}^{0}$ be the arc of $C$ from $a_{i-1}$ to $a_{i}$ in the counterclockwise direction. For each $i$ greater than one let $A_{i}^{1}, A_{i}^{2}, A_{i}^{3}, \cdots, A_{i}^{r_{i}-1}$ be $r_{i}-1$ independent arcs (numbered inwardly) spanning* $C$ between the points $a_{i-1}$ and $a_{i}$. Let these arcs all lie in the sector of the circle $C$ determined by the rays joining the origin to $a_{i-1}$ and $a_{i}$ and the portion of the circle swept out by the radius vector as it moves from $a_{i-1}$ to $a_{i}$ with increasing $\theta$. Construct these arcs in such a way that the length of $A_{i}^{j}$ is less than twice the length of $A_{i}^{0}$ for all $j \leqq r_{i}-1$. Also arrange that no radius of $C$ meets any $A_{i}^{j}$ in more than one point. We may then define $A_{i}^{n}$ for all positive integers $n$ by reducing the superscript $n$, modulo $r_{i}$. We define the limit set $L$ by the equation

$$
L=\sum_{i=1}^{\infty} \sum_{n=1}^{\infty} A_{i}^{n}
$$

For every positive integer $i$ define

$$
n_{i}=\sum_{j=1}^{i} 2^{i-j} r_{j} .
$$

We shall now construct an infinite sequence of point sets $P_{i}$ having the following properties: (i) $P_{i}$ consists of exactly $n_{i}$ points, for each $i$; (ii) $P_{i}$ lies wholly in the plane $S_{i}: z=1 / i, w=0$; (iii) $P_{i}$ converges to the set $L$ in the plane $z=0, w=0$.

Let $R_{i},(i=0,1,2, \cdots)$, denote the ray joining the origin to the point $a_{i}$, for each $i$. For $i$ fixed consider any two rays $R_{j-1}$ and $R_{j},(j \leqq i)$. Between these rays, but not including them, construct $2^{i-j}$ rays equally spaced in the sector and let them be named $K_{j}^{1}, K_{j}^{2}, K_{j}^{3}, \cdots, K_{j}^{2^{i-j}}$ in the counterclockwise order in which they occur. The ray $K_{j}^{n}$ will intersect $L$ in exactly $r_{j}$ points, one on each of the $\operatorname{arcs} A_{j}^{s},\left(s=0,1,2, \cdots, r_{j}-1\right)$. We arrange all these points of intersection, for $j=1,2, \cdots, i$, in a linear array $w_{1}, w_{2}, \cdots, w_{n_{i}}$, where $w_{t}$ is the point of intersection of the arc $A_{j}^{n}$ and the ray $K_{j}^{s}$ for the value

* An arc $a x b$ is said to span a point set $M$ provided $(a x b) M=a+b$. 


$$
t=n+1+(s-1) r_{j}+\sum_{m=1}^{j-1} 2^{i-m} r_{m} .
$$

It is easily seen that this orders the points first, according to which sector of the circle they are in, second, according to which ray they are on, and third, according to the free $\operatorname{arc}$ of $L$ which contains them. Let $w_{t}$ have the coordinates $\left(x_{t}, y_{t}, 0,0\right)$. Let $p_{i}^{t}$ be the point with coordinates $\left(x_{t}, y_{t}, 1 / i, 0\right)$, and define

$$
P_{i}=\sum_{t=1}^{n_{i}} p_{i}^{t}
$$

It follows easily that the sequence of point sets thus constructed satisfies (i), (ii), and (iii).

We have now reached the final stage of the construction which will be made by adding to $L$ an infinite sequence of 2 -cells, where each 2-cell is considered as the homeomorph of a triangle and its interior. It is thus meaningful to speak of the sides and vertices of a 2-cell. Every 2-cell will have one of its sides in $L$; this side will be called the base of the 2-cell. The other vertex of the 2-cell will lie in one of the planes $S_{i}$ and will be referred to as the apex of the 2-cell. By the altitude we mean the maximum distance from the base to any point of the 2-cell.

For every integer $i$ we shall construct a set of $n_{i} 2$-cells $G_{i}^{1}, G_{i}^{2}$, $G_{i}^{3}, \cdots, G_{i}^{n_{i}}$. The bases of the $G_{i}^{j}$ will be taken as the sets

$$
B_{i}^{j}=\sum_{k=1}^{i} A_{k}^{j}
$$

The apexes $b_{i}^{j}$ will be chosen to satisfy the following conditions: (a) all the $b_{i}^{j}$ are distinct; (b) for each $(i, j), G_{i}^{j} S_{i}=b_{i}^{j}$; (c) $\rho\left(b_{i}^{j}, p_{i}^{j}\right)$ $<1 / i$. We finally require that for every $\epsilon>0$ there exist at most a finite number of $G_{i}^{j}$ of altitude greater than $\epsilon$, and that the product of any of the 2-cells, which are not identical, lies in $L$. All of these conditions are easily satisfied in our four-dimensional space.

Our space $M$ is now defined to be

$$
M=L+\sum_{i=1}^{\infty} \sum_{j=1}^{n_{i}} G_{i}^{j}
$$

It is easily seen that $M$ is a locally connected continuum.

We must now define the transformation $T(M)=M$. To do this we define $T\left(A_{i}^{j}\right)=A_{i}^{j+1}$ as a homeomorphism having the end points of $A_{\imath}^{j}$ as fixed points. This defines $T$ on $L$. For each $G_{i}^{j}$ let $T\left(G_{\imath}^{j}\right)=G_{i}^{j+1}$ 
(where the superscript is reduced, modulo $n_{i}$, if necessary) be a homeomorphism agreeing with $T$ on $L$ and sending $b_{i}^{j}$ into $b_{i}^{j+1}$ (with the same convention on the superscripts). This defines $T$ for every $p$ of $M$. It is evident that $T(M)=M$ is a pointwise periodic homeomorphism.

If we now define

$$
G_{i}=\sum_{j=1}^{n_{i}} b_{i}^{j}
$$

we see that each $G_{i}$ is an orbit under $T$, and conditions (a) and (b) of the theorem are satisfied. The proof is thus complete.

University of PENNSYlvania AND

UNIVERSITY OF VIRGINIA

\section{AN ENUMERATION OF LOGICAL FUNCTIONS}

\section{WILLIAM WERNICK}

In a logical calculus of $m$ values, abbreviated by $L_{m}$, we may deal with functions of $n$ variables. A particular function is defined in this calculus if we assign a constant value, which may be any arbitrary one of the $m$ possible values in $L_{m}$, as the value of that function for a particular argument. It is the purpose of this note to enumerate, among all functions of $n$ variables in $L_{m}$ : those which depend on all $n$ variables in the argument; those which depend on just $(n-1)$ of the variables in the argument, being independent of one of them; and so on; finally those which are completely independent of all the variables in the argument.

Since each variable in the argument may assume values from $1, \cdots, m$, independently, there are $m^{n}$ possible arguments, and since to each argument we may assign independently, as a functional value, any of the $m$ values $1, \cdots, m$, there are in all $m^{m^{n}}$ possible functions of $n$ variables.

Let $V_{n}$ be the total number of all functions of $n$ variables in $L_{m}$. Then we have from the above

$$
V_{n}=m^{m^{n}} .
$$

Let $U_{n k}$ be the number of functions of $n$ variables which depend on exactly $k$ of them. (It is this expression for which we are seeking an explicit evaluation.) Since $k$ variables may be selected from $n$ of them in just $C_{n, k}$ ways, we have the relation:

$$
U_{n k}=C_{n, k} U_{k k} .
$$

